Enhancing User Interaction in Virtual Environments through Adaptive Personalized 3D Interaction Techniques

Peer-reviewed author version

OCTAVIA, Johanna; CONINX, Karin \& RAYMAEKERS, Chris (2010) Enhancing User Interaction in Virtual Environments through Adaptive Personalized 3D Interaction Techniques. In: DeBra, P; Kobsa, A; Chin, D (Ed.) USER MODELING, ADAPTATION, AND PERSONALIZATION, PROCEEDINGS. p. 423-426..

Handle: http://hdl.handle.net/1942/11052 


\title{
Enhancing User Interaction in Virtual Environments through Adaptive Personalized 3D Interaction Techniques
}

\author{
Johanna Renny Octavia \\ Supervisors: Karin Coninx and Chris Raymaekers \\ Hasselt University - tUL - IBBT \\ Expertise Centre for Digital Media \\ Wetenschapspark 2, 3590 Diepenbeek, Belgium
}

\begin{abstract}
Leveraging interactive systems by integrating adaptivity is considered as an important key to accommodate user diversity and enhance user interaction. A virtual environment is a highly interactive system which involves users performing complex tasks using diverse 3D interaction techniques. Adaptivity has not been investigated thoroughly in the context of virtual environments. This $\mathrm{PhD}$ research is concerned with embedding intelligence to enhance user interaction in virtual environments (i.e. providing adaptive personalized 3D interaction techniques).
\end{abstract}

Key words: virtual environments, adaptation, 3D interaction techniques

\section{Introduction and Research Problem}

Research on virtual environments has been growing vastly both in terms of quantities and areas of real applications, ranging from education to entertainment [1]. However, the issue of engaging users naturally and intuitively while they interact in a virtual environment still motivates researchers. In a virtual environment, users typically perform complex tasks with highly interactive 3D user interfaces and use a variety of $3 \mathrm{D}$ interaction techniques ranging from a simple technique to a very complex one [1]. This situation likely increases the complexity for users and eventually impedes them to interact naturally in the environment.

My $\mathrm{PhD}$ research is aimed to integrate adaptivity into virtual environments and investigate to what extent adaptivity can enhance natural and intuitive user interaction. This contribution details the research on integrating adaptive and personalized 3D interaction techniques for enhancing user interaction in virtual environments by means of intelligent algorithms.

\section{Research Approach}

We recognize the significance of enhancing user interaction in virtual environments by means of integrating intelligence and establishing adaptive personalized 
3D interaction techniques. At the start of this $\mathrm{PhD}$ research, we formulated a research approach to answer the challenge by proposing a conceptual framework for adaptive and personalized 3D interaction techniques as shown in Figure 1(a). Throughout the research, I am conducting a series of user experiments to construct and validate the various building blocks of the framework.

Using the proposed framework, we intend to gather information and build up knowledge about users' working methods, performances, preferences and abilities when performing tasks and interacting in a virtual environment. This knowledge will later on be used to assess the adaptation and personalization of interaction techniques with the help of intelligent algorithms. This $\mathrm{PhD}$ research focuses on investigating intelligent algorithms to provide three types of adaptation in virtual environments: (1) switching between interaction techniques, which offers the most suited interaction technique for a user in a certain situation, (2) adapting the interaction technique itself that is adjusting parameters of an interaction technique to control how the user should perform it, and (3) enhancing the interaction technique with modalities, which adds multimodal feedback, such as visual, audio, and force feedback into the technique in order to provide more control for the user.

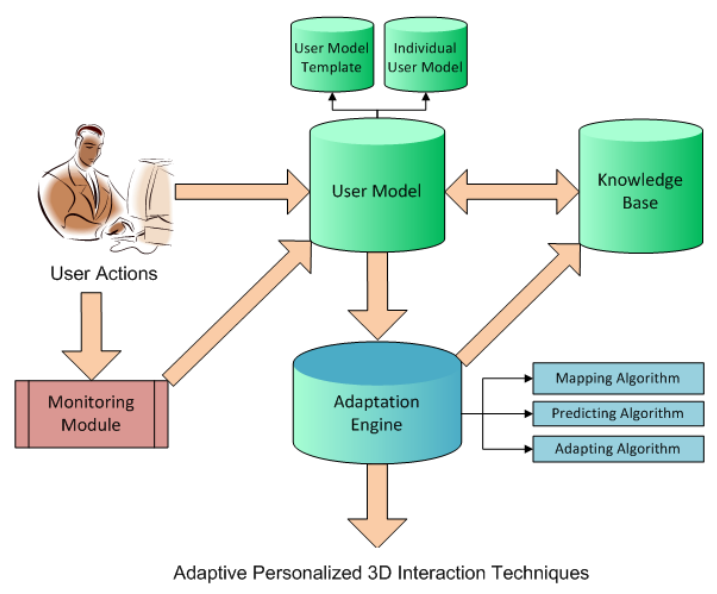

(a) Conceptual framework

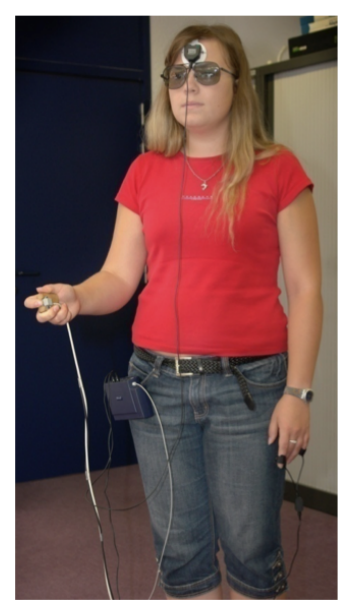

(b) Utilization of user's physiological measures

Fig. 1. Towards adaptive 3D interaction techniques in virtual environments

\section{Research Progress}

After conducting an exhaustive literature study on user modeling and adaptive and intelligent $(2 \mathrm{D} / 3 \mathrm{D})$ user interfaces, I started the realization of the conceptual 
framework by constructing the user model as the first building block. The user model, comprised of a user model template (general and group user models) and individual user models, is acquired by conducting user modeling activity through experiments. Two subsequent experiments were carried out with the objectives of constructing the user model template and establishing the individual user model. In both experiments, I focused on investigating user interaction when performing a 3D target acquisition task using two selection techniques, the bubble cursor and the depth ray [2].

The first experiment was conducted as an initial study to investigate the possibility of adaptation and personalization in virtual environments [3]. The experiment resulted in a general user model for 3D target acquisition tasks in virtual environments, which can be beneficial for novice users interacting in a virtual environment for the first time. In the second experiment, this user model template was verified to be favorable for enhancing first-time users' interaction in a virtual environment. Moreover, individual user models were successfully constructed which led to the establishment of the complete user model. After establishing the user models, I continued to the implementation of the adaptation engine as the next building block. The first type of adaptation, switching between interaction techniques, was implemented based on the user models and an algorithm built upon user's performance and preference of interaction technique.

In the second experiment, I also investigated the user's reaction to adaptation by gathering physiological data to assess user frustration with regard to adaptation. For this purpose, I utilized the ProComp Infiniti (see Figure 1(b)) to collect two kinds of physiological data: galvanic skin response (GSR) and electromyography (EMG). Concerning the adaptation of interaction technique implemented in the second experiment, I found that users perform significantly better and experience less frustration when adaptation is incorporated during their interaction in virtual environments [4].

\section{Future Work Plan}

The work completed to date has concentrated on investigating two building blocks of the framework, namely the user model and the adaptation engine. There are two more components of the framework left, the monitoring module and the knowledge base, that still need to be researched in the remaining time of the $\mathrm{PhD}$ research. The research will also continue to investigate further on the establishment of several intelligent algorithms that support the role of the adaptation engine.

Currently, I am preparing the conduct of an experiment to construct the monitoring module, where users' physiological measures are recognized and monitored as triggers for adaptation. I intend to investigate the utilization of user frustration as an indicator to provide adaptation in a gaming virtual environment. User frustration will be induced and measured throughout the game sessions and whenever frustration is detected, adaptive feedback will be provided with the expectation that it will decrease user frustration and increase user per- 
formance. The third type of adaptation, enhancing the interaction technique with modalities, will be implemented in the adaptation engine. The interaction technique will be adapted with the use of multimodal feedback such as visual and force feedback. For instance, particular users may perform a 3D target acquisition task better when the selection technique is complemented by haptics (force feedback) as such.

Following this experiment, longer-term goals include the investigation of the second type of adaptation (adapting the interaction technique itself) to be incorporated in the adaptation engine and also the investigation of intelligent interaction techniques. I would like to work on these ideas further on with a specific group of users (e.g. users with limited motor abilities).

\section{Conclusion and Expected Feedback}

This doctoral consortium paper mainly describes the research problem and work progress to date of my $\mathrm{PhD}$ research, which is currently situated at the intermediate stage. The motivation behind this $\mathrm{PhD}$ research is that I envision that by integrating adaptation and personalization into 3D interaction techniques, natural, intuitive and enhanced user interaction in virtual environments can be achieved. With this paper, I seek constructive feedbacks and suggestions for improvement particularly on these following issues:

1. The conceptual framework. How can we justify the comprehensiveness, accuracy, generality and applicability of the proposed framework, including each element that serves as building blocks of the framework?

2. The adaptation engine and intelligent algorithms. How to determine the effectiveness and efficiency of the adaptation engine? What aspects define the intelligence of such adaptation algorithms?

3. Adaptation determinants. What other aspects of user interaction in virtual environments that are considered significant in the adaptation process, besides user's performance, preference and physiological measures?

\section{References}

1. Bowman, D.A., Chen, J., Wingrave, C.A., Lucas, J., Ray, A., Polys, N.F., Li, Q., Haciahmetoglu, Y., Kim, J.S., Kim, S., Boehringer, R., Ni, T.: New directions in 3d user interfaces. International Journal of Virtual Reality 5(2) (2006) 3-14

2. Vanacken, L., Grossman, T., Coninx, K.: Multimodal selection techniques for dense and occluded 3d virtual environments. Int. J. Hum.-Comput. Stud. 67(3) (2009) $237-255$

3. Octavia, J.R., Raymaekers, C., Coninx, K.: Investigating the possibility of adaptation and personalization in virtual environments. In Houben, G., McCalla, G., Pianesi, F., Zancanaro, M., eds.: UMAP 2009. Volume 5535 of LNCS., Springer (2009) 361-366

4. Octavia, J.R., Raymaekers, C., Coninx, K.: Adaptation in virtual environments: Conceptual framework and user models. Multimedia Tools and Applications Journal accepted (2010) 1-20 